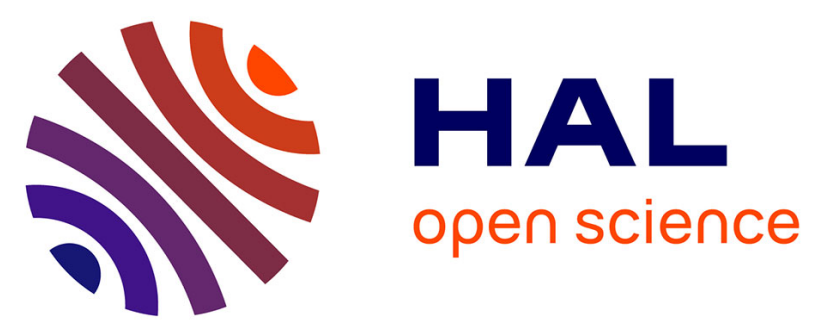

\title{
Adaptive Multi-Agent System and Mixed Integer Linear Programming Optimization Comparison for Grid Stability and Commitment Mismatch in Smart Grids
} Sharyal Zafar, Vishwajeet Maurya, Anne Blavette, Guy Camilleri, H Ben Ahmed, Marie-Pierre Gleizes

\section{To cite this version:}

Sharyal Zafar, Vishwajeet Maurya, Anne Blavette, Guy Camilleri, H Ben Ahmed, et al.. Adaptive Multi-Agent System and Mixed Integer Linear Programming Optimization Comparison for Grid Stability and Commitment Mismatch in Smart Grids. ISGT Europe, Oct 2021, Espoo (online), Finland. 10.1109/isgteurope52324.2021.9640019 . hal-03398611

\section{HAL Id: hal-03398611 \\ https://hal.science/hal-03398611}

Submitted on 23 Oct 2021

HAL is a multi-disciplinary open access archive for the deposit and dissemination of scientific research documents, whether they are published or not. The documents may come from teaching and research institutions in France or abroad, or from public or private research centers.
L'archive ouverte pluridisciplinaire HAL, est destinée au dépôt et à la diffusion de documents scientifiques de niveau recherche, publiés ou non, émanant des établissements d'enseignement et de recherche français ou étrangers, des laboratoires publics ou privés. 


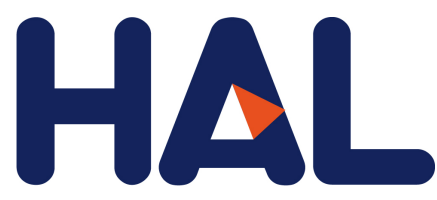

archives-ouvertes

\section{Adaptive Multi-Agent System and Mixed Integer Linear Programming Optimization Comparison for Grid Stability and Commitment Mismatch in Smart Grids}

S Zafar, V Maurya, Anne Blavette, G Camilleri, H Ahmed, M-P Gleizes

\section{To cite this version:}

S Zafar, V Maurya, Anne Blavette, G Camilleri, H Ahmed, et al.. Adaptive Multi-Agent System and Mixed Integer Linear Programming Optimization Comparison for Grid Stability and Commitment Mismatch in Smart Grids. ISGT Europe, Oct 2021, Espoo (online), Finland. hal-03398611

\section{HAL Id: hal-03398611 \\ https://hal.archives-ouvertes.fr/hal-03398611}

Submitted on 23 Oct 2021

HAL is a multi-disciplinary open access archive for the deposit and dissemination of scientific research documents, whether they are published or not. The documents may come from teaching and research institutions in France or abroad, or from public or private research centers.
L'archive ouverte pluridisciplinaire HAL, est destinée au dépôt et à la diffusion de documents scientifiques de niveau recherche, publiés ou non, émanant des établissements d'enseignement et de recherche français ou étrangers, des laboratoires publics ou privés. 


\section{Adaptive Multi-Agent System and Mixed Integer Linear Programming Optimization Comparison for Grid Stability and Commitment Mismatch in Smart Grids}

\author{
S. Zafar \\ SATIE \\ ENS-Rennes \\ Rennes, France \\ sharyal.zafar@ens-rennes.fr \\ G. Camilleri \\ IRIT \\ University of Toulouse \\ Toulouse, France \\ guy.camilleri@irit.fr
}

\author{
V. Maurya \\ IRIT \\ University of Toulouse \\ Toulouse, France \\ vishwajeet.maurya@grenoble-inp.fr \\ H. Ben Ahmed \\ SATIE \\ ENS-Rennes \\ Rennes, France \\ benahmed@ens-rennes.fr
}

\author{
A. Blavette \\ SATIE \\ CNRS, ENS-Rennes \\ Rennes, France \\ anne.blavette@ens-rennes.fr
M-P. Gleizes
IRIT
University of Toulouse
Toulouse, France
marie-pierre.gleizes@irit.fr

\begin{abstract}
Existing electrical networks are going through a transition and distributed energy resource, if not managed properly, can hinder this transition. Uncontrolled introduction of photovoltaics and electric vehicles in distribution networks would lead to substantial issues such as commitment mismatches, line congestions, voltage deviations, etc. This paper presents the use of a classical approach, mixed integer linear programming optimization, and a novel approach, adaptive multi-agent system, to solve the highlighted distribution side challenges by utilizing electric vehicles' storage capacity. This comparison serves as a great tool to benchmark the performance of the under-development adaptive multi-agent system methodology.
\end{abstract}

Keywords-Multi-Agent Systems, Mixed Integer Linear Programming, Optimality Gap, Electric Vehicles, Balance Responsible Party, Distribution Network Stability

\section{INTRODUCTION}

Electrical networks, at first designed for unidirectional power flows, are facing stability and resilience challenges due to introduction of modern distributed energy resources (DERs) such as photovoltaics (PVs) and electric vehicles (EVs). The European Union (EU) 2030 climate and energy framework [1] and projections by the International Energy Agency (IEA) [2] present a future with appreciable amount of such DERs connected to the distribution network. This certainly ensures a smoother path towards carbon-free energy systems but can also cause disruptions in the existing electrical infrastructure by bringing techno-economic challenges.

On the technical side, congestions in the network, voltage deviations as well as a need for energy balancing due to imperfect forecasts can occur. The financial responsibility of balancing is managed by Balance Responsible Parties (BRPs). BRPs submit a daily balance schedule to the Transmission System Operator (TSO) and manage energy balance within their portfolio called the Balance Perimeter (BP). This schedule is defined on a sub-hourly basis (soon to be harmonized to a quarter-hourly basis in Europe), known as the imbalance settlement period. If the BRP has consumed less energy (i.e., produced more energy) than what was planned, it would be compensated by the TSO and vice versa. To mitigate the commitment mismatch cost, a BRP may use, through an aggregator, flexibles entities (e.g., EVs). However, the BRP orders must not create local issues on the network, such as congestions. Indeed, the problem resides in the interactions between different actors managing different roles at different levels and having potentially contradictive objectives. Hence, with an increasing amount of DERs, it is necessary to mitigate these challenges.

Different solutions have been proposed to ensure an efficient coordination while maintaining grid stability. Grid reinforcement solutions are somewhat limited due to a significant infrastructure cost, therefore making flexibility solutions more promising. One such approach is the application of Multi-Agent System (MAS) concepts to manage networks. Hu et al. [3] have used swarm intelligence to model a MAS for controlling a distribution network with EVs. Lauri et al. [4] and Huang et al. [5] have designed MASs using Markov Decision Process (MDP) and game theory, respectively. A thorough study of existing literature suggests that most of the proposed MAS architectures can be classified as hierarchical, thus not fully decentralized. This property makes the system vulnerable to single point of failure (SPOF) issues and limits performance as sub-problems of limited size only, can be handled when strong real-time constraints are considered, which can be quite limiting for large-scale problems.

To overcome these challenges, a system based on a particular type of MAS, Adaptive Multi-Agent System (AMAS), is being developed. In AMAS, the goal is achieved through cooperative interactions of agents i.e., emergence. The main characteristics of such a system are autonomy, decentralization, sociability, and reactivity. The system is called ADaptive Energy Management In Smart Grids (ADEMIS) [6] and is capable of handling, by exploiting the storage capacity of EVs for ancillary services, basic grid stability challenges i.e., line congestions, as well as potential antagonist issues such as handling commitment mismatch while ensuring desired charging of the EVs.

It is equally important to keep a check of the obtained solutions' qualities, when developing a new methodology. For that purpose, the problem is simplified, modeled, and solved using Mixed Integer Linear Programming (MILP) optimization, which has already been used for optimal power 
flows in smart grids [7]. This enables to draw a comparison between a basic, uncoordinated charging strategy, the classical MILP optimization approach and the newly proposed AMAS methodology. A good comparison would allow us to better understand the position of AMAS solutions among different methodologies and quantify the room for improvements. Optimization formulation and linear, convex approximations for MILP along with AMAS modeling is presented in Section II. Following this, case studies are defined for testing in Section III and finally the results obtained are discussed in Section IV and conclusions are made.

\section{SYSTEM MODELING}

\section{A. Optimization Formulation}

The starting point is the power flow constraints of the network, which ensure power flow in the network based on its physical constraints. $P_{a}(t)$ and $Q_{a}(t)$ represent the active and reactive powers at bus $a$ at instant $t$. The power at each bus is equal to the sum of power injected or drawn from the bus. $P_{P l a n, E V, a}(t)$ and $\Delta P_{E V, a}(t)$ stand for the day-ahead planned EV power connected to bus $a$ at instant $t$, and the change in that power, hence a decision variable for optimization, to achieve the system's objective.

$$
\begin{aligned}
P_{a}(t)= & P_{a}^{\text {Generation }}(t)-P_{a}^{\text {Demand }}(t) \\
P_{a}(t)= & P_{\text {grid }, a}(t)+P_{P V, a}(t)-P_{\text {load }, a}(t) \\
& -\left(P_{\text {Plan }, E V, a}(t)+\Delta P_{E V, a}(t)\right) \\
Q_{a}(t)= & Q_{a}^{\text {Generation }}(t)-Q_{a}^{\text {Demand }}(t) \\
Q_{a}(t)= & Q_{\text {grid }, a}(t)+Q_{P V, a}(t)-Q_{\text {load }, a}(t) \\
& -\left(Q_{P l a n, E V, a}(t)+\Delta Q_{E V, a}(t)\right)
\end{aligned}
$$

Equation (5) links the power flows in the network to the bus voltages and network losses. $V_{a}$ stands for the voltage at bus $a$, while $Y_{a b}^{*}$ represents the conjugate of the admittance matrix. Equations (6) and (7) relate powers at the bus with total inflows and outflows. Equations (8) and (9) ensure that the power values remain within specified limits.

$$
\begin{gathered}
P_{a b}(t)+j Q_{a b}(t)=V_{a}(t)\left(V_{a}^{*}(t)-V_{b}^{*}(t)\right) Y_{a b}^{*} \\
\sum_{b} P_{a b}(t)=P_{a}(t) \\
\sum_{b} Q_{a b}(t)=Q_{a}(t) \\
P_{a, \text { min }} \leq P_{a}(t) \leq P_{a, \text { max }} \\
Q_{a, \text { min }} \leq Q_{a}(t) \leq Q_{a, \text { max }}
\end{gathered}
$$

Next comes the constraints on bus voltages and currents. These can be classified as Distribution System Operator (DSO) constraints.

$$
\begin{gathered}
V_{a, \min } \leq\left|V_{a}(t)\right| \leq V_{a, \max } \\
\left|I_{a b}(t)\right| \leq I_{a b, \max }
\end{gathered}
$$

EV constraints serve the purpose of modeling the dynamics of the EVs and also linking it with the network flow constraints. The State of Charge (SoC) of an EV is dependent on the previous state and charging or discharging power at the present interval. The SoC of the EV should be within specified limits, and it should be greater than the desired minimum SoC at the departure time. Similarly, the State of Health $(\mathrm{SoH})$ of an EV, models health of the battery and should never be negative. $P_{E V, \text { charging, } a}(t)$ and $P_{E V \text {,discharging, } a}(t)$ is the EV charging and discharging power connected to bus $a$ at instant $t$. Term $\rho(t)$ is a binary variable which makes sure that an EV is either charging or discharging, and not both, at interval $t$.

$$
\begin{aligned}
& S_{O} C_{a}(t)=S_{O} C_{a}(t-1)+\frac{P_{E V, \text { charging,a }}(t) \Delta t}{E_{\text {bat }}} \eta_{\text {charging }} \\
& -\frac{P_{E V, \text { discharging, } a}(t) \Delta t}{E_{\text {bat }}} \frac{1}{\eta_{\text {discharging }}} \\
& S O C_{\text {min }, a} \leq S O C_{a}(t) \leq S O C_{\max , a} \\
& S O C_{\text {min }, a}\left(t_{\text {departure }, a}\right) \leq S O C_{a}\left(t_{\text {departure }, a}\right) \\
& S O H_{E V, a}(t)=S O H_{E V, a}(t-1) \\
& -\frac{\left(P_{E V, \text { discharging, } a}(t)+P_{E V, \text { charging }, a}(t)\right) \Delta t}{0.2 E_{\text {bat }}} \\
& S_{\text {OH }, a}(t) \geq 0 \\
& P_{E V, a}(t)=P_{E V, \text { charging, } a}(t)-P_{E V, \text { discharging, } a}(t) \\
& =P_{\text {Plan } E V, a}(t)+\Delta P_{E V, a}(t) \\
& (1-\rho(t)) P_{\min , E V, a} \leq P_{E V, \text { discharging, } a}(t) \\
& \leq P_{\max , E V, a}(1-\rho(t)), \rho(t) \in\{0,1\} \\
& Q_{E V, a}(t)=P_{E V, a}(t) \tan \emptyset
\end{aligned}
$$

The objective is to minimize the mismatch cost paid by the BRP, (21). BRP is charged for a negative mismatch (i.e., over-consumption) and vice versa. The negative mismatch cost is higher compared to the compensation for a positive mismatch. Terms $C_{m i s}^{-}(T), C_{m i s}^{+}(T), E_{m i s}^{-}(T)$ and $E_{m i s}^{-}(T)$ represent the negative and positive mismatch costs and energy values, respectively. $P_{B R P}(t)$ is the real-time power consumption in the BRP perimeter, while $P_{\text {Plan }}(t)$ is the dayahead planned power defined by (25). PV forecasts are usually provided as a one-hour average value for distributed generation, while for loads the resolution is smaller i.e., 10 minutes. The errors in the forecasts are also modeled here.

$$
\begin{gathered}
\min _{\Delta P_{E V, a}(t)} C_{B R P}= \\
\min _{\Delta P_{E V, a}(t)} \sum_{T=1}^{T=T_{\text {end }}}\left(C_{\text {mis }}^{-}(T) E_{\text {mis }}^{-}(T)-C_{\text {mis }}^{+}(T) E_{m i s}^{+}(T)\right) \\
E_{\text {mis }}^{-}(T)= \\
\max \left(0, \sum_{t=T \Delta T}^{t=(T+1) \Delta T-\Delta t}\left(\left(P_{\text {Plan }}(T)-P_{B R P}(t)\right) \Delta t\right)\right) \\
E_{\text {mis }}^{+}(T)= \\
\max \left(0, \sum_{t=T \Delta T}^{t=(T+1) \Delta T-\Delta t}\left(\left(P_{B R P}(t)-P_{P l a n}(T)\right) \Delta t\right)\right) \\
P_{B R P}(t)=\sum_{a} P_{a}(t) \\
\left(1+\operatorname{err}_{\text {load }}(t)\right)-\operatorname{mean}_{10 \text { min }}\left(P_{E V a}(t)\left(1+\operatorname{err}_{E V}(t)\right)(25)\right. \\
P_{P l a n}(T)=\frac{1}{\Delta T} \sum_{t=T \Delta T}^{t=(T+1) \Delta T-\Delta t} \operatorname{mean}_{1 h r}
\end{gathered}
$$

\section{B. MILP Approximations}

To linearize the original non-linear and non-convex problem, (6) is approximated, obtained by applying small angle approximation $\left(\sin \left(\theta_{a}-\theta_{b}\right)=\theta_{a}-\theta_{b}\right)$, (26)-(27). Also, the subtraction of the positive energy mismatch cost (a convex function), in the objective function makes the curvature of the total objective function non-convex. To retain the convexity, the positive mismatch cost has been considered as $0 € / \mathrm{MWh}$, which represents a worst-case.

$$
\begin{aligned}
& P_{a b}(t)= G_{a b}\left(V_{a}(t)-V_{b}(t)\right)+B_{a b}\left(\theta_{a}(t)-\theta_{b}(t)\right) \\
& Q_{a b}(t)=B_{a b}\left(V_{a}(t)-V_{b}(t)\right)+G_{a b}\left(\theta_{b}(t)-\theta_{a}(t)\right) \\
& \min _{\Delta P_{E V, a}(t)} C_{B R P}= \\
& \min _{\Delta P_{E V, a}(t)} \sum_{T=1}^{T=T}\left(C_{\text {mis }}^{-}(T) E_{\text {mis }}^{-}(T)\right)
\end{aligned}
$$




\section{ADEMIS Modeling}

This work is a continuation of Blanc-Rouchossé et al. [6]. The basic architecture of the system is the same as described in the previous work. Each physical component of the distribution grid is modeled as a cooperative agent, with each agent having its own objective, a partial information of the environment and a set of actions depending on the situation. The AMAS system executes in a loop. During each iteration, an agent calculates its own criticality, between 0 and 1 , and compare it to the criticalities of the neighbouring agents. Subsequently it decides to take an action, either to satisfy its own objective or to help the neighbouring agents in achieving theirs. In the following paragraphs, the modelling of each type of agent is defined.

1) Line Agent: Line agents communicate criticalities, (29)(30), based on the line current values. Term $I_{t h}$ is the threshold value at which line becomes critical and is set to 0.8 times the rated line current value $I_{\max } . C r_{a, l}$ is the anticipated criticality of the line to ensure dynamics of the system, so that the line does not keep on oscillating between highly critical and non-critical states. Once a line becomes congested, its line agent will keep on communicating the anticipated criticality value so EV agents can continue cooperating with the line without disturbing the dynamics of the system.

$$
\begin{gathered}
C r_{l}(t)=\frac{\left(I(t)-I_{t h}\right)}{\left(I_{\max }-I_{t h}\right)} ; I_{t h}=0.8 I_{\max }, \text { if } I \geq I_{t h} \\
C r_{a, l}(t)=C r_{a, l}(t-1)+K_{l}\left(C r_{l}(t)-C r_{a, l}(t-1)\right)
\end{gathered}
$$

2) BRP Agent: BRP agent sends its criticality, (31)-(32), based on its instantaneous mismatch value. Terms $K_{l}$ and $K_{B R P}$ are tuning parameters with arbitrarily selected values 0.01 and 100 , respectively.

$$
\begin{gathered}
\operatorname{Cr}_{B R P}(t)=\frac{|\Delta E(t)|}{t_{\text {remaining,period } K_{B R P}}} \\
|\Delta E(t)|=\sum_{i=t_{\text {start }}\left(P_{B R P}(i)-P_{\text {Plan }}\right) \Delta t}^{t}
\end{gathered}
$$

3) EV Agent: $\mathrm{EV}$ agents calculate their respective criticalities based on (33). In (33), $P_{\max }$ is the maximum charging or discharging power of each EV, $t_{\text {remaining,departure }}$ is the time remaining before departure of the EV. After this calculation, they compare it to the criticality signals they have received from the neighbouring agents. Based on this, each EV agent makes the decision, either to help a line for congestion, or the BRP for commitment mismatch or to continue charging, by calculating the updated power for that interval. The updated power is calculated using (34). $\mathrm{Cr}_{\text {max }}$ stands for the maximum criticality received while $\mathrm{Cr}_{\max , 2}$ is the second maximum criticality among antagonist (opposite) signals

$$
\begin{gathered}
C r_{E V}(t)=\frac{\left(\operatorname{SOC}_{\min }-\operatorname{SOC}(t)\right) E_{\text {bat }}}{P_{\text {max }} t_{\text {remaining,departure }}} \\
P_{E V}(t)=P_{E V}(t-1) \pm \\
\left(C r_{\text {max }}(t)-h * C r_{\text {max }, 2}(t)\right) P_{\text {max }}
\end{gathered}
$$

In the above equation, $h$ is a function which tackles the situation when EV agents receives antagonistic requests. For example, if line is congested (thus requesting EVs to charge less) and BRP commitment mismatch is positive (thus creating favourable conditions for EVs to charge more) at a given instant simultaneously, then EV agents have to decide which request is more critical. Thus, the $h$ function acts like a weighting function, given in (35).

$$
h\left(C r_{a, l}\right)=\left\{\begin{array}{c}
1+\frac{\alpha-1}{T_{l, \min }} C r_{a, l}, \text { if } C r_{a, l}<T_{l, \min } \\
\exp \left(\frac{C r_{a, l}}{K_{a}+K_{b}}\right), \text { if } T_{l, \min } \leq C r_{a, l} \leq T_{l, \max } \\
1-\frac{\beta}{1-T_{l, \max }} C r_{a, l}, \text { if } C r_{a, l}>T_{l, \max }
\end{array}\right.
$$

$T_{l, \max }$ and $T_{l, \min }$ are thresholds for anticipated line criticality. Between two thresholds, the $h$ parameter behaves exponentially and linearly otherwise. Terms $K_{a}$ and $K_{b}$ are two parameters dependent on values of $\alpha, \beta, T_{l, \max }$ and $T_{l, \text { min }}$. The development of the methodology for ADEMIS being at an early stage, it can be observed that a simple strategy consisting in reducing the commitment mismatch was selected at first, thus not considering the potential reward for injecting power when there is an excess of PV production. However, the goal of this paper consists in assessing the room for improvement with such a usually envisaged strategy among BRPs, as well as to check that constraints are satisfied.

\section{CASE STUdY}

\section{A. Distribution Network}

The "IEEE Low Voltage Test Feeder" (IEEE LVTF) [8] is selected for this case study. As shown in Fig. 1, it consists of 3 zones (A, B \&C). It is connected to a substation through a $11 \mathrm{kV} / 416 \mathrm{~V}$ transformer. The network consists of 55 households. These 55 household buses are also equipped with a PV and an EV connection, making the size of total EV fleet for this study equals to 55. For a congestion study, line 117 (highlighted in red) of the network is selected. For ADEMIS power flow, the network is simulated in DIgSILENT PowerFactory (PF).

\section{B. Datasets}

This study is carried out for a total time of one hour (4 imbalance settlement periods of 15 minutes each), from 16:00 till 17:00. The dataset for load profiles of 55 households is provided along with the IEEE LVTF model. The resolution of the data is 1 minute. The irradiance data for PV profiles (resolution of 1 second) is obtained from the database of the National Renewable Energy Laboratory (NREL) [9]. The power generated by a panel is calculated using (36). $\operatorname{Irr}(t)$ is the irradiance at time instant $t, A$ is the area of the panel, 10 $m^{2}$ here, $\eta_{P V}$, the efficiency of the panel, is equal to 0.17 . The error for day-ahead PV forecast is set to $15 \%$ during the studied hour.

$$
P_{P V}(t)=A \eta_{P V} \operatorname{Irr}(t)
$$

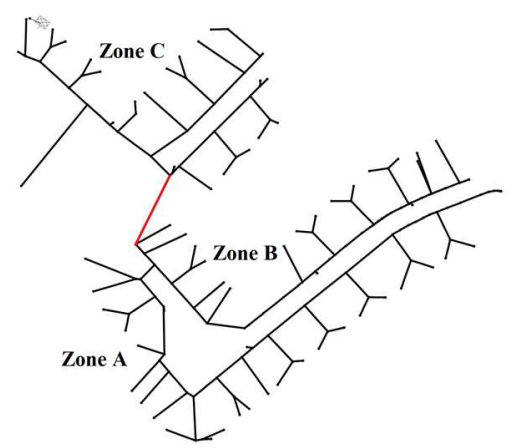

Fig. 1. Graphical representation of the IEEE low voltage test feeder 
The EV fleet modeled for this system can be classified into three distinct types of EVs, 1) EVs arriving at start of period 1 for charging and departing soon, which would create a congestion in the network line 117, 2) EVs arriving during the simulation time and departing near the end or after simulation time, and 3) EVs connected to the network before the start of the simulation and departing afterwards. All EVs present the same capacity of $30 \mathrm{kWh}$ and have a maximum charging or discharging power of $7 \mathrm{~kW}$. Terms $S o C_{\min }$ and $S o C_{\max }$ have been set to 0.3 and 0.8 respectively, while $S o C_{\min }\left(t_{\text {departure }}\right)$ is equal to 0.7 .

Additional data for imbalance costs of the BRP is obtained through the French TSO Réseau de Transport d'Électricité (RTE) [10]. The data provided by the TSO has a resolution of 30 minutes, thus a linear resampling of data is done to obtain costs values for each 15 minutes period. Negative mismatch cost values are 91.26, 108.83, 103.28 and $92.124 € / \mathrm{MWh}$, while positive mismatch values are $82.56,98.46,93.44,89.35$ $€ /$ MWh for periods 1, 2, 3 and 4, respectively.

\section{Antagonist Issues}

This study is modeled to incorporate a variety of scenarios to make it more realistic and non-trivial. During period 1, PVs are over-producing, while under-producing in the remainder. BRP mismatch is present in all 4 periods, due to differences in the forecasted and real-time values. Also, during period 1 a line congestion is expected when uncoordinated charging occurs. The rated value for line current is $0.065 \mathrm{kA}$. The line type (number of cores and cross-sectional area) is also given with the IEEE LVTF model. The rated current value is obtained from the technical sheet [11] for the congested line. To provoke a congestion in the network, $8 \mathrm{EVs}$ in zone $\mathrm{A}$ and 4 EVs in zone B are simulated to arrive near the start of the simulation. A summary is presented in Fig. 2.

EVs causing congestion are charging at their maximum specified power as their departure time is around 16:12. As the EVs load forecast is made by taking the average over 10 minutes-periods, if the total load is very high (enough to cause congestion) for a portion of 10 minutes and very low for the remainder, the average value would be in the middle and would not depict congestion for the portion when a very high total load is present, which is exactly what happening in this scenario. As stated, we have over-production and congestion in period 1, which gives rise to an antagonistic scenario, where EVs may be incentivized to consume more power but as there is a line congestion, line agent would require EVs to draw lesser power through the line.

\section{RESULTS}

In this section, first the results obtained from MILP and ADEMIS are discussed individually and then a comparison of both along with the basic charging strategy is made.

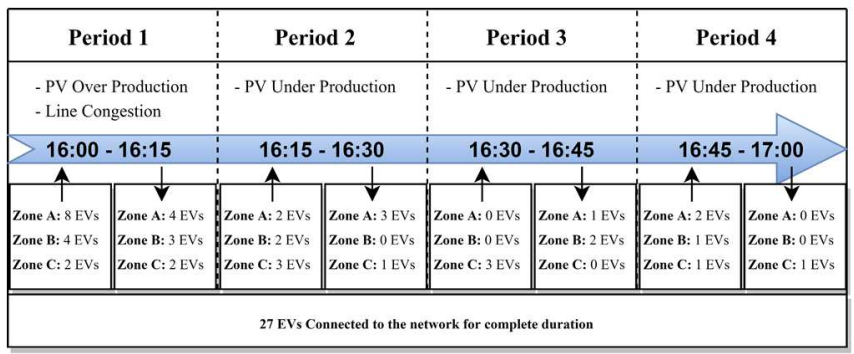

Fig. 2. Summary of the case study, EVs arriving ( $\uparrow)$ and departing $(\downarrow)$

\section{A. MILP Results}

To model MILP formulation CVXPY [12], with GUROBI solver, is used. The computing machine is equipped with Intel i5-10210U CPU with base speed of $2.11 \mathrm{GHz}$ and 8 GB RAM (DDR4). The temporal resolution of the study is 1 minute. Fig. 4-b shows that during period 1, when congestion is expected to occur with an uncoordinated charging, the total EV consumption is lower than the forecasted consumption. Power flow from the grid to the congested zones is reduced, and the EVs present in both zones, which do not need to depart early, started supporting the EVs which are departing soon by charging less or discharging.

To check the impact of the approximations on the quality of the solution, the optimized power profiles obtained through MILP are simulated in the IEEE LVTF PowerFactory model. Active power grid profiles are shown in Fig. 3-a. The percentage cosine similarity between both curves is $99.018 \%$. This confirms a minor impact of MILP approximations on the quality of the solution. To draw a fair comparison, battery aging cost has been omitted in the MILP formulation, as it is not present in the current version of ADEMIS. This results in a significant number of oscillations in the solution profiles.

\section{B. ADEMIS Results}

Agents' behaviour is implemented in JAVA. Power flow for each iteration is done in PowerFactory. Both platforms communicate through a python script. As the agents react to the system in real-time, the time step considered for each iteration is 1 second. The system consists of $55 \mathrm{EV}$ agents.

Fig. 4-a confirms that the congestion in the network is also avoided for ADEMIS solution as the line current is smaller than the rated current value of the line. Present version of ADEMIS is configured to reduce the commitment mismatch, as explained in (31-32). Further, the EVs present in both zones, which do not need to depart soon, are helping the EVs departing soon by discharging.

\section{Results Comparison}

Fig. 4-a shows the line currents in line 117. Both ADEMIS and MILP solutions are successful in managing the line congestion, which should have occurred with a basic charging strategy. Further, both for MILP and ADEMIS, the SoC criteria (minimum SoC reached before EV departure) of all the EVs are satisfied, which cannot be guaranteed for the basic charging approach in case a zone(s) gets disconnected from the grid side due to congestion or other failures in the network.

A comparison of energy mismatch for each period is presented in Fig. 5. This comparison is between different formulations presented earlier, obtained through different methodologies to tackle the same set of problems. Negative values indicate positive energy mismatch (over-production), whereas positive values mean negative energy mismatch (over-consumption).

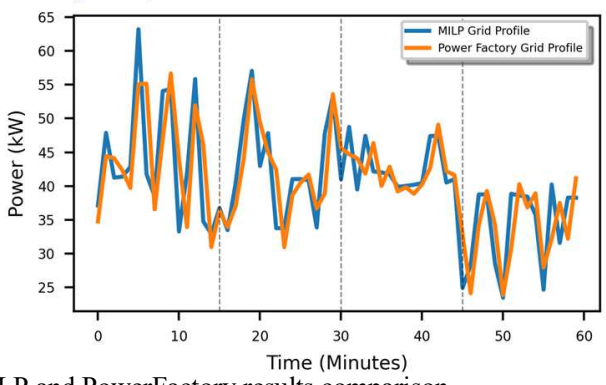

Fig. 3. MILP and PowerFactory results comparison 

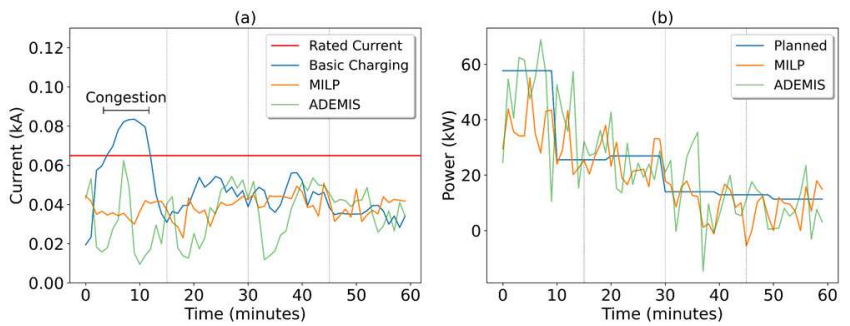

Fig. 4. Results comparison: a) Line 117 current b) Total EV powers

For periods 2,3 and 4, ADEMIS and MILP follow the same trend of significantly reducing the expected negative energy mismatch, compared to the uncoordinated charging strategy. In period 1, the cost of injecting more power and battery aging is zero in this preliminary study. Hence, the MILP approach proposes one feasible solution among others, where a large amount of power is injected in the grid (at no cost). However, using the non-convex objective function, this injection is rewarded. Under these conditions, the behaviour of ADEMIS is counterproductive compared to the basic charging, as it reduces the injection of power into the external grid. The goal of studying the conditions in period 1 was primarily to check that the EVs and DSO constraints were satisfied when antagonistic requests were received by the agents, knowing that the basic strategy implemented in the agents would not be relevant in this particular case. More sophisticated BRP functions are considered for future work in order to improve the performance of the overall system.

Finally, the cost comparison for each formulation is done by placing the obtained solution values in (21) (for MILP both (21) and (28) are used) and then calculating ratios with MILP PF results as reference. Again, it can be verified, in Table I., that the solution obtained through ADEMIS stands between MILP and basic charging strategy. MILP, (28), solution is closer to the ADEMIS solution, as in neither of the approaches the exploitation of the reward by injecting power is sought.

\section{CONCLUSION}

A comparison of MILP optimization and AMAS has been presented to solve the grid stability and commitment mismatch problems in the distribution grid. Both approaches efficiently handle the given challenges. Compared to the basic charging strategy, ADEMIS solution is performing better overall, but a substantial gap between ADEMIS and MILP solutions indicates that there is large room for improvement.
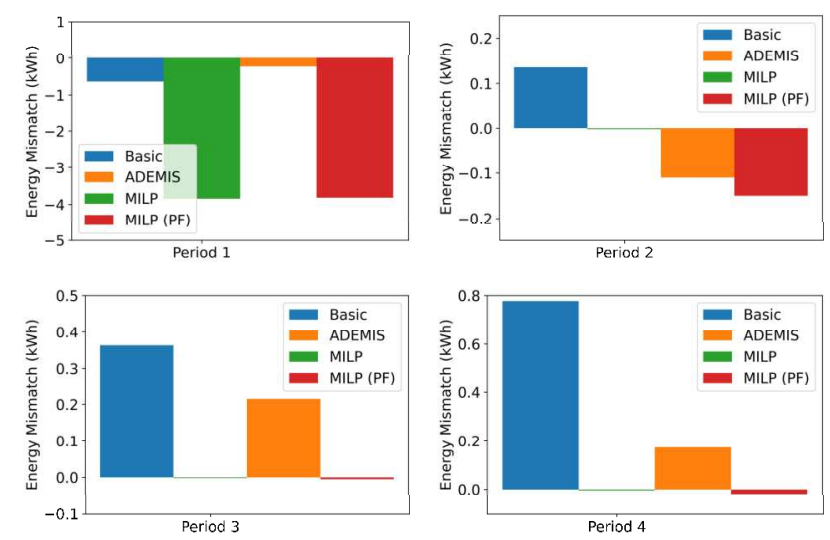

Fig. 5. Total energy mismatch comparison for each period
TABLE I. COST COMPARISON (RATIO) OF STUDIED FORMULATIONS

\begin{tabular}{|l|l|c|c|c|c|}
\hline \multirow{2}{*}{$\begin{array}{c}\text { Ratio } \\
\left(\frac{x}{x_{\text {ref }}}\right)\end{array}$} & \multicolumn{5}{|c|}{ Strategy } \\
\cline { 2 - 6 } & Basic & ADEMIS & $\begin{array}{c}\text { MILP } \\
(\mathbf{2 8})\end{array}$ & $\begin{array}{c}\text { MILP } \\
(\mathbf{2 1})\end{array}$ & $\begin{array}{c}\text { MILP } \\
(\boldsymbol{P F})\end{array}$ \\
\hline Period 1 & 0.165 & 0.063 & 0 & 1.006 & 1 \\
\hline Period 2 & -0.933 & 0.733 & 0 & 0 & 1 \\
\hline Period 3 & -37 & -22 & 0 & 0 & 1 \\
\hline Period 4 & -36 & -8 & 0 & 0 & 1 \\
\hline Total & -0.21 & -0.02 & 0 & 0.95 & 1 \\
\hline
\end{tabular}

On the flip side, AMAS is more modular and scalable compared to the MILP. Anticipative stochastic optimization is based on forecasts which can have errors and requires a large computing time, being NP-complete. Unlike this approach, an AMAS system reacts to the environment in real-time and the computing time of each agent is independent of total number of agents in the system. This provides motivation for the future work, which includes improving the behaviour of the agents as well as bus voltage management to study large-scale unbalanced networks. This would give a better benchmark to compare not only the quality of the solutions but also the time complexity to obtain those solutions.

\section{ACKNOWLEDGMENT}

This work is carried out as part of the "Opt-Real" project funded by the Brittany Regional Council which is gratefully acknowledged.

\section{REFERENCES}

[1] 2030 climate \& energy framework, [Online]. Available: https://ec.europa.eu/clima/policies/strategies/2030_en

[2] IEA Renewables 2020, [Online]. Available: https://www.iea.org/reports/renewables-2020/solar-pv

[3] Junjie Hu, Hugo Morais, Morten Lind, Henrik W. Bindner, "Multiagent-based modelling for electric vehicle integration in a distribution network operation", Electric Power Systems Research, vol. 136, pp. 341-351, ISSN 0378-7796, 2016.

[4] F. Lauri, G. Basso, J. Zhu, R. Roche, V. Hilaire \& A. Koukam, (2013). Managing Power Flows in Microgrids Using Multi-Agent Reinforcement Learning, Agent Technologies for Energy Systems.

[5] H. Huang, Y. Cai, H. Xu, and H. Yu, "A Multiagent Minority-GameBased Demand Response Management of Smart Buildings Toward Peak Load Reduction", IEEE Transactions on Computer-Aided Design of Integrated Circuits and Systems, vol. 36, no. 4, pp. 573-585, April 2017. DOI: https ://doi.org/10.1109/TCAD.2016.2571847

[6] Blanc-Rouchosse, Jean-Baptiste \& Blavette, Anne \& Ben Ahmed, H. \& Camilleri, Guy \& Gleizes, Marie-Pierre. (2019). Multi-Agent System for Smart-Grid Control with Commitment Mismatch and Congestion, [Online]. Available: 10.1109/ISGTEurope.2019.8905760.

[7] G. Liu, M. Starke, Xiaohu Zhang and K. Tomsovic, "A MILP-based distribution optimal power flow model for microgrid operation," 2016 IEEE Power and Energy Society General Meeting (PESGM), 2016, pp. 1-5, [Online]. Available: 10.1109/PESGM.2016.7741704.

[8] IEEE Distribution Test Feeders, May 2019, [Online]. Available: http://sites.ieee.org/pestestfeeders/

[9] Sengupta, M.; Andreas, A. (2010). Oahu Solar Measurement Grid (1 YearArchive): 1-Second Solar Irradiance; Oahu, Hawaii (Data); NREL Report No.DA-5500-56506, [Online]. Available: http://dx.doi.org/10.5439/1052451.

[10] Réseau de Transport d'Électricité (RTE) balancing costs, [Online]. Available: https://www.services-rte.com/fr/visualisez-les-donneespubliees-par-rte/equilibrage.html

[11] Low voltage power cables, technical information, pp. 12-27, [Online]. Available: http://www.bahra-cables.com/downloads/low_voltage.pdf

[12] Steven Diamond, et al. "CVXPY: A Python-embedded modeling language for convex optimization". Journal of Machine Learning Research, vol. 17, no. 83, pp. 1-5, 2016. 